\title{
Present State and Prospects for the Meteor Research in Ukraine
}

\author{
O. Shulga ${ }^{1}$, Y. Voloshchuk ${ }^{2}$, S. Kolomiyets ${ }^{2}$, Y. Cherkas ${ }^{2}$, \\ I. Kimakovskay ${ }^{3}$, S. Kimakovsky ${ }^{3}$, E. Knyazkova ${ }^{3}$, Y. Kozyryev ${ }^{4}$, \\ Y. Sybiryakova ${ }^{4}$, Y. Gorbanev ${ }^{3}$, I. Stogneeva ${ }^{3}$, V. Shestopalov ${ }^{3}$, \\ P. Kozak ${ }^{5}$, O. Rozhilo ${ }^{5}$ and Y. Taranukha ${ }^{5}$
}

\author{
${ }^{1}$ Nikolaev Astronomical Observatory, email: avshulga@mail.ru \\ ${ }^{2}$ Kharkiv National University of Radioelectronics, ${ }^{3}$ Astronomical observatory of I.I.Mechnikov \\ Odessa National University, ${ }^{4}$ Nikolaev Astronomical Observatory, ${ }^{5}$ Astronomical Observatory \\ of Kyiv National Taras Shevchenko University
}

Abstract. ODESSA. Systematical study of the meteor events are being carried out since 1953 In 2003 complete modernization of the observing technique was performed, and TV gmeteor patrolh on the base of WATEC LCL902 cameras was created. @ wide variety of mounts and objectives are used: from Schmidt telescope $\mathrm{F}=540 \mathrm{~mm}, \mathrm{~F} / \mathrm{D}=2.25$ (field of view $\mathrm{FOV}=$ $(0.68 \times 0.51) \mathrm{deg}$, star limiting magnitude SLM = $13.5 \mathrm{mag}$, star astrometric accuracy 1-2 arcsec) up to Fisheye lenses $\mathrm{F}=8 \mathrm{~mm}, \mathrm{~F} / \mathrm{D}=3.5(\mathrm{FOV}=(36 \mathrm{x} 49) \mathrm{deg}, \mathrm{SLM}=7 \mathrm{mag})$. The database of observations that was collected between 2003 and 2012 consists of 6176 registered meteor events. Observational programs on basis and non-basis observations in Odessa (Kryzhanovka station) and Zmeiny island are presented. Software suite of 12 programs was created for processing of meteor TV observations. It enables one to carry out the whole cycle of data processing: from image preprocessing up to orbital elements determination. Major meteor particles research directions: statistic, areas of streams, precise stream radiant, orbit elements, phenomena physics, flare appearance, wakes, afterglow, chemistry and density. KYIV. The group of meteor investigations has been functioning more than twenty years. The observations are carried out simultaneously from two points placed at the distance of $54 \mathrm{~km}$. Super-isocon low light camera tubes are used with photo lens: $\mathrm{F}=50 \mathrm{~mm}, \mathrm{~F} / \mathrm{D}=1.5(\mathrm{FOV}=(23.5 \times 19.0) \mathrm{deg}, \mathrm{SLM}=9.5 \mathrm{mag})$, or $\mathrm{F}=$ $85, \mathrm{~F} / \mathrm{D}=1.5(\mathrm{FOV}=(13 \times 11) \mathrm{deg}, \mathrm{SLM}=11.5 \mathrm{mag})$. Astrometry, photometry, calculation of meteor trajectory in Earth atmosphere and computation of heliocentric orbit are realized in developed gFalling Starh software. KHARKOV. Meteor radio-observations have begun in 1957. In 1972, the radiolocation system MARS designed for automatic meteor registration was recognized as being the most sensitive system in the world. With the help of this system 250 000 faint meteors (up to $12 \mathrm{mag}$ ) were registered between 1972 and 1978 (frequency $31.1 \mathrm{MHz}$, particle masses $10^{-3} \sim 10^{-6} \mathrm{~g}$ ). Simultaneously, millions of reflections were registered for even fainter meteors (up to $14 \mathrm{mag}$ ). Information about 250000 meteors and 5160 meteor streams is included in database. This is an unique material that can be used for hypotheses testing, as well as for creation new theories about meteor phenomena. Models of the meteor matter distribution in the Earthfs atmosphere, near-Earth space and in the Solar system, influence on surface of spacecrafts were developed. NIKOLAEV. The optical and radio observations of meteors have begun in 2011. Two WATEC LCL902 cameras are used with photo lens $\mathrm{F}=85 \mathrm{~mm}, \mathrm{~F} / \mathrm{D}=$ $1.8(\mathrm{FOV}=(3.2 \times 4.3), \mathrm{SLM}=12 \mathrm{mag}$, star astrometric accuracy 1-6 arcsec $)$. Original software was developed for automatic on-line detection of meteor in video stream. During 2011 year 105 meteor events were registered (with angular length (0.5-4.5) deg and brightness (1-5) mag). Error of determination of the meteor trajectory arc $\sim(10-12)$ arcsec. Error of determination of the large circle pole of the meteor trajectory is $\sim(3-13)$ arcmin. In the radio band observations of meteors are performed by registration of signal reflected from the meteor wake. As a signal source the over-the-horizon FM station in Kielce (Poland) is used. Narrow-beam antenna, computer with TV/FM tuner and audio recording software are used to perform radio observations. Original software was developed for automatic detection of meteor in audio stream. 